\title{
Mathematical biomarkers for the autonomic regulation of cardiovascular system
}

\section{Luciana A. Campos ${ }^{1 *}$, Valter L. Pereira Jr ${ }^{1}$, Amita Muralikrishna ${ }^{2}$, Sulayma Albarwani ${ }^{3}$, Susana Brás ${ }^{4}$ and Sónia Gouveia ${ }^{4}$}

${ }^{1}$ Center of Innovation, Technology and Education-(CITE), Camilo Castelo Branco University (UNICASTELO), Sao Jose dos Campos, Brazil

2 Department of Automation and Systems Technology, Federal Institute of Science and Technology of Sao Paulo, Sao Jose dos Campos, Brazil

${ }^{3}$ Department of Physiology, Sultan Qaboos University, Muscat, Oman

${ }^{4}$ Institute of Electronics and Telematics Engineering of Aveiro, University of Aveiro, Aveiro, Portugal

\section{Edited by:}

Geoffrey A. Head, Baker IDI Heart and Diabetes Institute, Australia

\section{Reviewed by:}

Alessandro Silvani, Università di

Bologna, Italy

Elena Lukoshkova, Russian

Cardiology Research and Production

Complex, Russia

\section{${ }^{*}$ Correspondence:}

Luciana A. Campos, Center of

Innovation, Technology and

Education-(CITE), Camilo Castelo

Branco University (UNICASTELO),

Sao Jose dos Campos Technology

Park, Presidente Dutra Road Km

138, Sao Jose dos Campos, SP

12247-004, Brazil

e-mail: camposbaltatu@yahoo.com
Heart rate and blood pressure are the most important vital signs in diagnosing disease. Both heart rate and blood pressure are characterized by a high degree of short term variability from moment to moment, medium term over the normal day and night as well as in the very long term over months to years. The study of new mathematical algorithms to evaluate the variability of these cardiovascular parameters has a high potential in the development of new methods for early detection of cardiovascular disease, to establish differential diagnosis with possible therapeutic consequences. The autonomic nervous system is a major player in the general adaptive reaction to stress and disease. The quantitative prediction of the autonomic interactions in multiple control loops pathways of cardiovascular system is directly applicable to clinical situations. Exploration of new multimodal analytical techniques for the variability of cardiovascular system may detect new approaches for deterministic parameter identification. A multimodal analysis of cardiovascular signals can be studied by evaluating their amplitudes, phases, time domain patterns, and sensitivity to imposed stimuli, i.e., drugs blocking the autonomic system. The causal effects, gains, and dynamic relationships may be studied through dynamical fuzzy logic models, such as the discrete-time model and discrete-event model. We expect an increase in accuracy of modeling and a better estimation of the heart rate and blood pressure time series, which could be of benefit for intelligent patient monitoring. We foresee that identifying quantitative mathematical biomarkers for autonomic nervous system will allow individual therapy adjustments to aim at the most favorable sympathetic-parasympathetic balance.

Keywords: heart rate variability, cardiovascular system, mathematical modeling, fuzzy logic, nonlinear dynamics, linear models, baroreflex

\section{INTRODUCTION}

According to the World Health Organization (2011), hypertension and cardiovascular events continue to constitute the leading global diseases affecting more than $20 \%$ of the world's population (Harris, 2011). Although very important progress in the treatment and prevention of these diseases has been found, intense contemporary research efforts are aiming to unravel new diagnostic tools. Accumulating experimental evidence indicates that markers of autonomic nervous system such as heart rate variability (HRV) may contribute to cardiovascular diagnosis and have prognostic value (Asl et al., 2008; Ramirez-Villegas et al., 2011).

A biomarker generally refers to a key molecular or cellular event that is linked to a health outcome. Biomarkers are quantifiable and objective features of biological processes (Lee et al., 2007). Biomarkers may detect specific disease stages and processes allowing individual therapy adjustments. The development of mathematical algorithms as biomarkers to describe biological processes represents an active research area of quantitative biology and medicine. Blood pressure and heart rate are vital signs characterized by a high degree of temporal variability that is controlled by neurohumoral factors. The autonomic nervous system plays an important role in the regulation of blood pressure and heart rate through variations in its sympathetic and parasympathetic activity (Kezdi and Geller, 1968; Gootman and Cohen, 1970). Heart rate and blood pressure variability have frequently been used as biomarker of sympatho-vagal balance (Pagani et al., 1986). Sympathetic and parasympathetic systems activities are regulated through baroreflex mechanisms that tightly control blood pressure and heart rate. The baroreflex sensitivity (BRS) is a measure of baroreflex function and is defined as alterations in beat-to-beat interval (milliseconds) per unit change in blood pressure ( $\mathrm{mm} \mathrm{Hg}$ ). BRS is influenced by various neuroendocrine systems, including central renin angiotensin system (Campos et al., 2004, 2006a) and melatonin (Campos et al., 2013b). Interplay between these systems might be responsible for the circadian alterations in cardiovascular function (Baltatu et al., 2002; Campos et al., 2006b, 2013a). 
BRS may be determined pharmacologically through injection of vasoactive substances (such as phenylephrine and sodium nitroprusside) and invasive determination of arterial pressure (Campos et al., 2013b). Non-invasive methods for BRS evaluation may be through measurement of heart rate/blood pressure changes in response to deep breath, Valsalva maneuver (forced expiration against resistance), or tilt testing (passive body movement from a supine position to an upright tilt). These tests provide an index of cardiovagal function of baroreflex (Levin, 1966; Wheeler and Watkins, 1973; Borst et al., 1982; Cooke et al., 1998; Shields, 2009). However, they may be altered by some factors, including rating and depth of breathing, position of the subject, and the presence of some diseases, such as diabetic neuropathy (Sundkvist et al., 1982; Low, 1993). Continuous monitoring of heart rate offers the possibility of evaluating the spontaneous adaptations of BRS as result of dynamic variations of blood pressure (Baltatu et al., 2001; Gouveia et al., 2009). Spectral analysis of blood pressure and HRV is applied to evaluate spontaneous BRS (Head et al., 2001). Low and high frequency spectral components of the blood pressure oscillations are related with the frequency oscillations in R-R interval due to baroreflex activity (La Rovere et al., 2008). These methods for BRS evaluation have different clinical implications such as in diagnostic and clinical management of cardiovascular diseases (La Rovere et al., 2008).

\section{HEART RATE VARIABILITY}

Heart rate can be defined as the number of cardiac cycles per unit of time. Cardiac cycle includes a period of relaxation (diastole) and a period of contraction (systole) of the heart, representing the time period necessary for the given event to repeat itself. The time interval between two successive $\mathrm{R}$ waves in the electrocardiogram (ECG) equals one cardiac cycle. Thus, the ECG can measure heart rate through the recording of electrical potentials generated by the heart's electrical activity. The frequency $(f)$ is the inverse of the period (T), thus, $f=1 / \mathrm{T}$. HRV is the variation of beat-to-beat intervals, also known as R-R intervals. The HRV indexes are obtained by analyzing the intervals between $\mathrm{R}$ waves, which can be captured by instruments including electrocardiograph, digital-to-analog converter and cardio frequency meter from surface electrodes that are placed at specific points on the body (Rajendra Acharya et al., 2006).

Time domain and frequency domain are two types of methods commonly used to analyze cardiovascular variability. Both methods apply to linear data structure. Time domain uses continuous monitoring of cardiovascular parameters while frequency domain uses spectral analysis to express heart rate oscillation (Task force, 1996).

\section{TIME DOMAIN INDEXES OF CARDIOVASCULAR VARIABILITY}

Linear HRV analysis in time domain employs statistical methods. Data needs to be normalized before analysis. In order to make a comparison between different data sets, these have to be acquired over similar periods of time. The mostly used periods of time are $24 \mathrm{~h}$ (long term) and 5-30 min (short term). The time domain indexes are based on normal sinus beat-to-beat intervals (normalto-normal, or NN), and the most commonly used are: (a) standard deviation of all NN intervals (SDNN, milliseconds; Table 1). Its values depend on the length of the recording data: longer the length higher SDNN values. Therefore, a comparison of SDNN values of different length may lead to inappropriate interpretation (Task force, 1996). Low SDNN values are a predictor of high mortality in cardiovascular diseases (Kleiger et al., 1987; Task force, 1996; Nolan et al., 1998);

(b) root mean square of the successive differences (RMSSD, milliseconds; Table 1). It is an indication of short-term HRV components (Task force, 1996), reflects parasympathetic activity and is correlated with sudden death in epilepsy (DeGiorgio et al., 2010) and fibrillation (Dash et al., 2009).

(c) adjacent successive NN intervals differing more than $50 \mathrm{~ms}$ (NN50; Table 1) and its percentage (pNN50). It indicates short-term HRV components and reflects parasympathetic activity (Task force, 1996). The values of NN50 have been correlated with autonomic neuropathy in diabetic patients (Ewing et al., 1991).

(d) triangular index, calculated from the number of all NN intervals divided by the maximum of the density distribution (Table 1). Estimate the overall HRV over $24 \mathrm{~h}$ (Task force, 1996) and it is influenced mainly by low frequencies (Malik et al., 1989).

(e) triangular interpolation of $\mathrm{NN}$ interval histogram (TINN; Table 1). It represents the baseline width of the distribution measured as a base of a histogram triangle approximating the RR interval distribution (Malik and Camm, 1993; Vanderlei et al., 2009). Estimate overall HRV over $24 \mathrm{~h}$ (Task force, 1996) and it is influenced mainly by low frequencies (Malik et al., 1989).

\section{FREQUENCY DOMAIN INDEXES OF CARDIOVASCULAR VARIABILITY}

Linear HRV analysis in frequency domain employs mathematical algorithms for frequency assignment. Physiological data collected as a time series can be considered as a sum of sinusoidal oscillations with different frequencies. The conversion of time domain analysis to frequency domain can be done through a mathematical transformation developed nearly two centuries ago (1807) by a French mathematician named Jean Baptiste Joseph Fourier (1768-1830). This process, called spectral analysis, allows signal decomposition originated from time series (tachogram) in its different frequency components, or in the so-called frequency bands. Noteworthy is that frequency refers to the number of times that a particular phenomenon occurs related to time. Typically, the unit for frequency is Hertz $(\mathrm{Hz})$, which is equivalent to one cycle per second.

From continuous recordings of heart rate, the total power (TP) is decomposed into three distinct bands in humans:

1. high frequency band (HF; Table 1) with frequency ranging from 0.15 to $0.40 \mathrm{~Hz}$, related to the heart rate variations associated with the respiratory cycle, commonly called respiratory sinus arrhythmia. It is usually modulated by the parasympathetic nervous system (Kuusela et al., 2003; Rajendra Acharya et al., 2006). HF is also known as "respiratory" band because 
Table 1 | Time and frequency domain measures of heart rate variability (Kamen and Tonkin, 1995; Task force, 1996; Brennan et al., 2001).

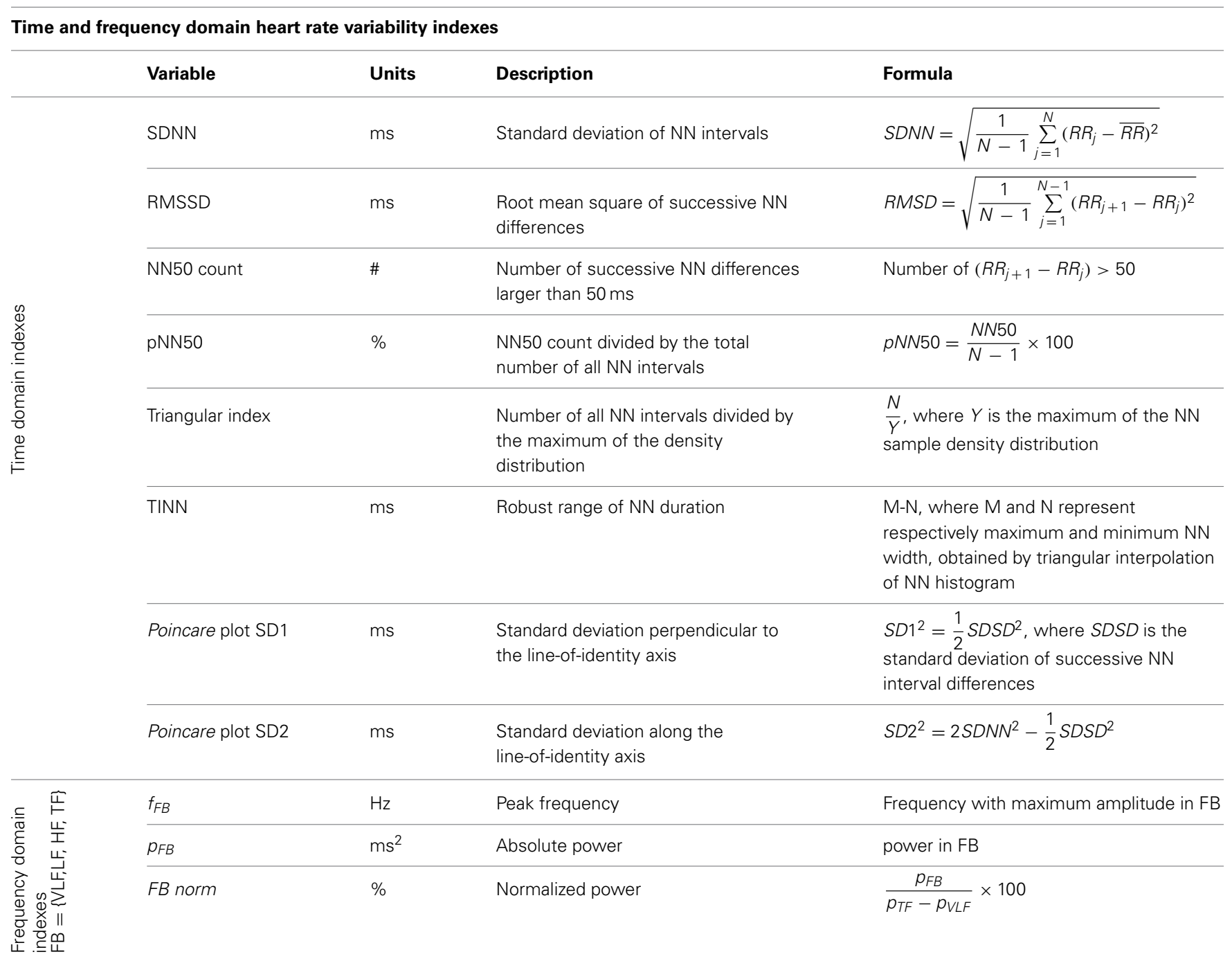

$F B$, frequency bands; VLF, very low frequency; $L F$, low frequency; $H F$, high frequency; $T F$, total frequency.

it corresponds to the R-R fluctuations caused by breathing (Bernardi et al., 1989; Ori et al., 1992; Bernardi et al., 1995);

2. low frequency band (LF; Table 1) with frequency ranging from 0.04 to $0.15 \mathrm{~Hz}$ is modulated by both sympathetic and parasympathetic nervous systems (Kuusela et al., 2003; Rajendra Acharya et al., 2006);

3. very low frequency band (VLF; Table 1) with frequency ranging from 0.003 to $0.04 \mathrm{~Hz}$, it is a variable which depends on the renin-angiotensin system, whose regulation is also affected by sympathetic and parasympathetic nervous systems (Taylor et al., 1998; Kuusela et al., 2003; Acharya et al., 2004). The lower frequency of the VLF band depends on window length. It was demonstrated an association of VLF with mortality after cardiac infarction (Bigger et al., 1992).

In the analysis of fetal heart rate (FHR) tracings, these frequency bands are usually adjusted to VLF $(0-0.03 \mathrm{~Hz}), \operatorname{LF}(0.03-0.15 \mathrm{~Hz})$, and HF $(0.5-1 \mathrm{~Hz})$, and hold the same physiological associations as in human adults. FHR recordings additionally exhibit a MF frequency band related to the fetal movements and maternal breathing (Signorini et al., 2003).

These frequency bands are not applicable for smaller animals such as rats and mice. In rats, the components of VLF to HF of HRV power spectra vary between 0.0 and $3.0 \mathrm{~Hz}$ (Task force, 1996; Baltatu et al., 2001; Bezerra et al., 2001; Ushizima et al., 2001; Silva et al., 2009), while in mice, the components of LF to HF vary between 0.1 and $5.0 \mathrm{~Hz}$ (Ishii et al., 1996; Joaquim et al., 2004; Baudrie et al., 2007).

Measurement of spectral components is typically made in absolute power $\left(\mathrm{ms}^{2}\right)$. However, the values of LF and HF can also be expressed in normalized units (NU) representing the value of each component relative to the TP minus the VLF component (Task force, 1996).

Both time and frequency domain methods for variability evaluation analyze linear properties of the data, as described above. These methods have limitations, as they require windowing of the 
data and it uses deterministic algorithms that are valid only to periodic phenomena. Therefore, there is a need to identify a hierarchy of models, each suited for a different type of investigation or to different parts of the system and devise strategies to couple them, using a multiscale framework. Systems of differentialalgebraic equations can be employed to study systemic behavior and auto-regulation mechanisms.

Heart period and blood pressure are coupled in a closed loop. The coupling between heart period and blood pressure via baroreflex regulation can be evaluated using cross-correlation analysis (Silvani et al., 2008, 2011), regression analysis by the events technique (Gouveia et al., 2009) and causal estimates (Acharya et al., 2004; Porta et al., 2011). Cross-correlation analysis gives a correlation coefficient between variations of heart period and blood pressure (Silvani et al., 2011). Geoffrey Head et al. demonstrated that cross-spectral coherence transfer function between heart period and blood pressure could be used effectively to reproducibly estimate BRS (Head et al., 2001). The transfer function gives information about coherence, gain and phase relation of both signals (Baltatu et al., 2001). Coupling between heart period and systolic blood pressure in healthy subjects varies among wake-sleep states (Silvani et al., 2008). It was suggested that cross-correlation function is applicable to study the balance between central autonomic and baroreflex control of heart rate. For instance, there are positive and negative correlations between heart period and blood pressure values that result from baroreflex and central autonomic controls, respectively (Silvani et al., 2011). However, in cases of BRS impairment, cross-correlation is likely to be statistically not significant, due to autonomic balance alterations. To address this shortcoming, the so called events technique based on baroreflex events, was proposed to maximize simultaneously the number of beats considered for BRS estimation and the correlation between the corresponding systolic blood pressure and $\mathrm{RR}$ values, in order to provide a more accurate baroreflex slope estimate (Gouveia et al., 2009). This technique has shown to accurately detect baroreflex alterations, associated with postural changes (Gouveia et al., 2009) and druginduced BRS stimulation (Beloka et al., 2009). This technique has also been demonstrated to be capable of providing segments of short and of long beat length, which were associated with parasympathetic and the sympathetic ANS activities, respectively (Gouveia et al., 2010).

One of the disadvantages is that cross-correlation and linear regression methods are able to estimate only the linear component of heart period and systolic blood pressure coupling (Silvani et al., 2008; Gouveia et al., 2009). The causal relationship theory between heart period and systolic blood pressure was introduced by Oppenheim (Oppenheim and Schafer, 1975). This led to studies to find the dominant causal direction in the interactions between heart period and systolic blood pressure. Cross-conditional entropy was used to study heart pulse-systolic blood pressure causality during head-up tilt test in heart transplanted patients, and compared with classical approach of linear methods. The head-up tilt induced progressive shift from the prevalent causal direction to the reverse causality in healthy subjects (Porta et al., 2011). Although cross-conditional entropy is a tool to determine the causality, it is unable to account for the combined exogenous influences of respiration on R-R interval and systolic blood pressure variability (Baselli et al., 1994; Cooke et al., 1999; Westerhof et al., 2006; Porta et al., 2011).

\section{NON-LINEAR INDEXES OF CARDIOVASCULAR VARIABILITY}

The nonlinear theory has been growing among physiologists and physicians aiming to explain the workings of biological phenomena, highly complex, dynamic, and interdependent, where the system behavior differs from the behavior of its parts or elements (Huikuri et al., 2003).

The exponent of power-law, approximate entropy (ApEn) analysis and detrended fluctuation (DFA) are nonlinear methods recently introduced to the study of HRV.

Entropy is a measure of randomness or disorder, as included in the second law of thermodynamics, namely the entropy of a system that tends toward the maximum. Different states of a system tend to evolve from ordered configurations to less organized settings, but statistically more likely. Referring to the time series analysis, the ApEn provides a measure of the degree of irregularity or randomness within a series of data. Entropy was originally used by Pincus (1991) as a measure of system complexity, where smaller values indicate greater regularity, and higher values lead to more disorder, randomness, and complexity of the system. For instance with a drop in the ApEn, heart rate becomes more regular with age in both men and women (Ryan et al., 1994).

The DFA is a technique that characterizes the variation pattern through measuring scales. DFA has been specifically developed to distinguish between intrinsic fluctuations generated by the complex system and those caused by external or environmental stimuli acting on the system (Peng et al., 1995). The variations that arise due to extrinsic stimulation are presumed to cause a local effect, while the intrinsic variations due to the dynamics of the system are assumed to exhibit a long-term correlation.

The analysis of the Poincare plot or Lorenz plot is considered as based on nonlinear dynamics by some authors (Kamen and Tonkin, 1995; Voss et al., 2007; Vanderlei et al., 2010). The Poincare plot is a two-dimensional graphical representation of the correlation between consecutive RR intervals, where each interval is plotted against the next one (Lerma et al., 2003), and its analysis can be done qualitatively (visually) by evaluating the shape formed by its attractor, which shows the degree of complexity of the RR intervals (Woo et al., 1992), or quantitatively, by fitting an ellipse to the figure formed by the plot from where the indexes are taken: SD1, SD2, and SD1/SD2 ratio (Tulppo et al., 1996; Vanderlei et al., 2010). SD1 represents the dispersion of points perpendicular to the line of identity and appears to be an index of instantaneous beat-to-beat variability (i.e., the short-term variability which is mainly caused by respiratory sinus arrhythmia), while the SD2 represents the dispersion of points along the line of identity and it characterizes long-term HRV. The SD1/SD2 ratio shows the relationship between short and long-term RR interval variations (Gamelin et al., 2006; Rajendra Acharya et al., 2006). Despite the fact that Poincaré plot is primarily considered a nonlinear technique, it has been shown that SD1 and SD2 can be obtained as a combination of linear time domain HRV indexes (Brennan et al., 2001, Table 1). Therefore, alternative measures 
are still needed to characterize nonlinear features in Poincaré plot geometry.

\section{FUZZY LOGIC CONCEPTS}

The possibility of using mathematical methods and theories for data analysis has opened up a range of possibilities for the study of pathophysiological behaviors of cardiovascular variability ( $\mathrm{Hu}$ et al., 2009; Sassi et al., 2009; Gieraltowski et al., 2013). Large volume of data can be more easily assessed and analyzed with fuzzy logic. In order to better understand the onset and development of important pathologies, the autonomic nervous system activity can be explored through dynamical fuzzy logic models, such as the discrete-time model and the discrete-event model. Fuzzy logic approaches are able to perform non-linear mapping or predictions involving more than one cardiovascular parameter and to explore possible relations among these parameters, which normally would not be considered as a possibility. Fuzzy logic represents a flexible system that adequately describes nonlinear and complex systems since the resulting function can be written as a weighted linear combination of the system inputs and, therefore, it can resemble a nonlinear function as needed. For this reason, fuzzy logic methods are a feasible solution to consider in the absence of prior mathematical description between input-output variables (Kovacic and Bogdan, 2006).

Considering the Sugeno Fuzzy Logic formulation, the system output $\mathrm{z}$ can be modeled from

$$
z=\frac{\sum_{i=1}^{N} w_{i} z_{i}}{\sum_{i=1}^{N} w_{i}},
$$

where $N$ corresponds to the number of fuzzy rules and $z_{i}=$ $\sum_{j=1}^{n} a_{i} x_{j}+c_{i}$ is a linear combination of the system inputs $x_{j}, j=$ $1, \ldots n$. The rule weights are obtained as $w_{i}=\prod_{j=1}^{n} \Gamma_{F_{j}^{i}}\left(x_{j}\right)$ where $\Gamma_{F_{j}^{i}}$ is the membership function of rule $i$ and input $x_{j}$. Although membership functions may assume different shapes, the Gaussian function is rather a popular choice in the literature due to its symmetry and dependence on mean and variance, which correspond respectively to the center and the width of the membership function.

Fuzzy logic has the singular characteristic to combine empirical knowledge (described as linguistic rules) and knowledge directly extracted from the data (Sadegh-Zadeh, 1999), enabling an easier way to interpret the outcomes in a physiological perspective. This mathematical model may be a reliable method to evaluate the influence of the autonomic nervous system over cardiovascular control in healthy and diseased subjects (Carvalho et al., 2002).

The main advantage of the use of fuzzy logic systems comes from their power to deal adequately with the uncertainty (Zadeh, 1975; Kovacic and Bogdan, 2006). In particular, this approach tolerates imprecise data, and it is focused on the "plausibility" of occurrence rather than the traditional binary response " 0 " or " 1 ."
For example, while a given measurement of a certain biological variable such as stress may convey a person as being "content," the same measurement may reveal a status of "dissatisfaction" for another one. Thus, biological variables that vary from person to person and are closely influenced by external and internal changes direct themselves toward fuzzy logic model of analysis, where the application of methods of investigation based on zero and one, true and false does not apply (Zadeh, 1975). Cardiovascular signals are characterized by a great intra- and inter-individual variability, besides imprecise measurements due to limited resolution of acquisition systems. Additionally, it is believed that traditional statistical methods may not capture all the information needed to describe disease in its complexity and dynamics (Grossi, 2005). In this context, fuzzy logic may be a more reliable alternative to traditional methods.

\section{APPLICATIONS OF FUZZY LOGICS TO THE ANALYSIS OF CARDIOVASCULAR VARIABILITY}

Fuzzy logic approaches have been recently used in the cardiovascular field in different contexts including applications in signal processing and monitoring, classification, prediction or control. One approach consists of extracting the relevant features from one or more cardiovascular signals, which are then integrated into a fuzzy logic scheme aiming at the identification of the presence or the quantification of a pathological state.

Fuzzy logic methods have been successfully integrated in control systems. For instance during anesthesia, mean arterial pressure was controlled based on the error between desired and measured values, allowing it to control the balance between the unconsciousness and the side effects caused by the hypnotic drug (Meier et al., 1992). Also during anesthesia, hemodynamic changes were successfully modeled considering drug dose level alterations as inputs of the fuzzy system (Nunes and Amorim, 2008). In hemodialysis condition, fuzzy logic has also shown to be capable of effectively control blood pressure trends, using ultra-filtration rate as input (Mancini et al., 2007). Such a system allowed an overall reduction of $40 \%$ of the most severe episodes in hypotension-prone subjects.

Abnormal cardiac rhythms have been identified using artificial neural network and fuzzy interactions based on nonlinear heart period R-R features, such as spectral entropy, Poincare SD1/SD2, and Lyapunov exponent (Acharya et al., 2004). Also based on R-R features, fuzzy logic was used for ECG beat classification to detect arrhythmic and ischemic heartbeats (Tsipouras et al., 2007). Fuzzy logic approaches showed efficiency in improving oscillometric cuff pressure measurements by properly detecting outliers and noise artifacts (Lin et al., 2003).

With the goal of evaluating autonomic nervous system function, fuzzy logic has been used to choose the optimum subset of time, frequency and nonlinear variables related to sympathetic and parasympathetic activities on HRV (Petkovic et al., 2013). Fuzzy logic approach has been used in a classification scheme to jointly evaluate results of several autonomic tests, e.g., head-up tilt test and active postural change, using both time and spectral analysis of heart rate and of diastolic blood pressure series (Carvalho et al., 2002). Similar fuzzy logic schemes were used for the information fusion of 
relevant features extracted from multimodal cardiovascular signals, such as heart period R-R and systolic blood pressure, for the detection of life threatening states in cardiac care units (Kannathal et al., 2006).

Recently, fuzzy logic methods have been employed to effectively describe blood pressure and heart period R-R coupling and, therefore, have the potential to improve time domain BRS estimation (Liu et al., 2008; Gouveia and Bras, 2012). The autoregressive linear analysis approach for BRS estimation has limitations when cardiovascular regulation is depressed. Liu et al. proposed a hybrid model consisting of a parallel modular structure with an autoregressive and a fuzzy logic system, to study simultaneously linear and non-linear heart rate and blood pressure coupling mechanisms (Liu et al., 2008). This approach illustrates the utility of combining more traditional methods with fuzzy logic, which could be of advantage in diseased conditions when cardiovascular system regulation is afflicted.

Time domain BRS methods based on spontaneous data typically assume blood pressure and heart period R-R linearity and provide single slope estimation, regardless of the blood pressure value (Beloka et al., 2009; Gouveia et al., 2009). In this context, fuzzy logic methods can contribute to establish a BRS dependent of blood pressure level, similarly to time domain blood pressure pharmacological methods. Recently, fuzzy logic has been used to analyze spontaneous R-R series as a function of blood pressure values, comparing performances in real and surrogate data (Gouveia and Bras, 2012). As an illustrative example, Figure 1 shows fuzzy logic curves and the membership functions for a healthy subject. The fuzzy logic curve obtained from real data is much less flatter than that obtained from isodistribution surrogate data (gained by shuffling the RR data) and exhibits significantly lower average modeling errors (Figure 1A). Also, the non-uniform location of the membership functions suggests that different systolic blood pressure values contribute differently to RR modeling (Figure 1B). These results indicate that fuzzy logic is able to model R-R changes connected to blood pressure alterations, besides the mean value and, thus, has the potential to improve time domain BRS estimation in spontaneous conditions. It remains to be assessed the clinical impact of these findings and inherent repercussion on BRS estimation. Finally, Figure 2 illustrates the flexibility of fuzzy logic-based modeling in the identification of heterogeneous shapes and patterns in systolic blood pressure and RR association for a set of several subjects.

Given the complexity of the mechanisms regulating heart rate and its non-linear characteristics, it is reasonable to assume that HRV analysis based on non-linear methods would generate valuable knowledge on the systems involved in the HRV regulation. The mainly used non-linear method of HRV analysis is Poincaré plot, which although simple and easy to use, it does not reflect the number of samples at each point of the graph, leading to errors of judgment of its plots (Hnatkova et al., 1995). The use of fuzzy logic and the consequent use of a "plausibility" rather than a binary logic may help to overcome the drawbacks found in the traditional methods of analysis.
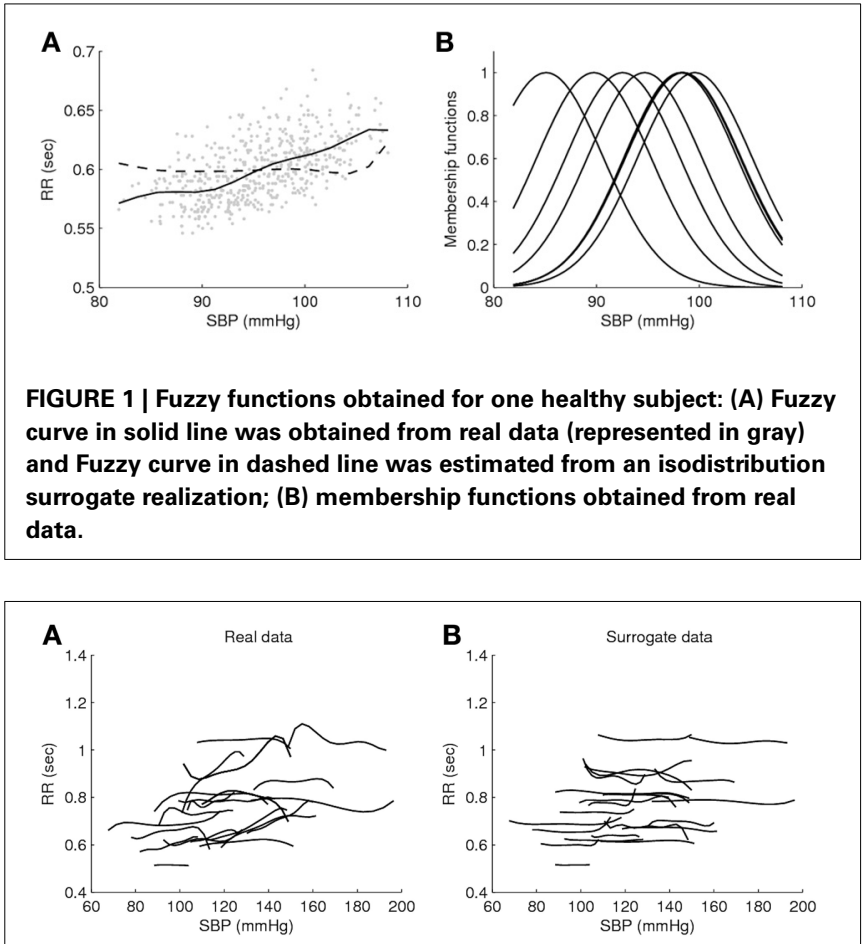

FIGURE 2 | Fuzzy surfaces obtained for a set of 23 subjects in Lying condition [EuroBaVar dataset (Gouveia and Bras, 2012)], with a curve in each subplot according to one subject. Subplot (A) shows the fuzzy curves obtained from real data and subplot (B) shows the fuzzy curves estimated from an isodistribution surrogate data.

\section{CONCLUDING REMARKS}

Fuzzy logic is a suitable choice when the system deals with uncertainty data, when linguistic interpretation is needed and when data "plausibility" should be taken into account. Fuzzy logic has been successfully used in different scenarios for the analysis of cardiovascular variability, with special emphasis on control and classification systems. Recent studies point out fuzzy logic also as a modeling alternative for cardiovascular time series, with potential impact on the estimation of joint parameters, e.g., arterial BRS. Therefore, fuzzy logic is a promising approach for the analysis of cardiovascular system and its regulatory mechanisms in normal and diseased conditions.

We expect an increase in accuracy of modeling and a better estimation of the heart rate and blood pressure time series, which could be of benefit for intelligent patient monitoring. We foresee that identifying quantitative mathematical biomarkers for autonomic nervous system will allow individual therapy adjustments to aim at the most favorable sympathetic-parasympathetic balance.

\section{ACKNOWLEDGMENTS}

This review was partially supported by São Paulo Research Foundation (Grants 2013/06698-0, 2013/50137-2), European Regional Development Fund (FEDER) through the programme COMPETE and by the Portuguese Government through the 
FCT, Fundação para a Ciência e a Tecnologia, in the scope of the projects FCOMP-01-0124-FEDER-022682 (FCT ref. PEst-C/EEI/UI0127/2011), Incentivo/EEI/UI0127/2013 (Instituto de Engenharia Electrónica e Telemática de Aveiro, IEETA/UA, Aveiro, www.ieeta.pt), and Cloud Thinking

\section{REFERENCES}

Acharya, R., Kumar, A., Bhat, P. S., Lim, C. M., Iyengar, S. S., Kannathal, N., et al. (2004). Classification of cardiac abnormalities using heart rate signals. Med. Biol. Eng. Comp. 42, 288-293. doi: 10.1007/BF02344702

Asl, B. M., Setarehdan, S. K., and Mohebbi, M. (2008). Support vector machine-based arrhythmia classification using reduced features of heart rate variability signal. Artif. Intell. Med. 44, 51-64. doi: 10.1016/j.artmed.2008.04.007

Baltatu, O., Afeche, S. C., Jose dos Santos, S. H., Campos, L. A., Barbosa, R., Michelini, L. C., et al. (2002). Locally synthesized angiotensin modulates pineal melatonin generation. J. Neurochem. 80, 328-334. doi: 10.1046/j.0022-3042.2001.00701.x

Baltatu, O., Janssen, B. J., Bricca, G., Plehm, R., Monti, J., Ganten, D., et al. (2001). Alterations in blood pressure and heart rate variability in transgenic rats with low brain angiotensinogen. Hypertension 37, 408-413. doi: 10.1161/01.HYP.37.2.408

Baselli, G., Cerutti, S., Badilini, F., Biancardi, L., Porta, A., Pagani, M., et al. (1994). Model for the assessment of heart period and arterial pressure variability interactions and of respiration influences. Med. Biol. Eng. Comput. 32, 143-152. doi: 10.1007/BF02518911

Baudrie, V., Laude, D., and Elghozi, J. L. (2007). Optimal frequency ranges for extracting information on cardiovascular autonomic control from the blood pressure and pulse interval spectrograms in mice. Am. J. Physiol. Regul. Integr. Comp. Physiol. 292, R904-R912. doi: 10.1152/ajpregu.00488.2006

Beloka, S. P., Gouveia, S., Gujic, M., Naeije, R., Rocha, A. P., and van de Borne, P. (2009). Differential effects of oral beta blockade on cardiovascular and sympathetic regulation. J. Cardiovasc. Pharmacol. Ther. 14, 323-331. doi: 10.1177/1074248409350137

Bernardi, L., Bianchini, B., Spadacini, G., Leuzzi, S., Valle, F., Marchesi, E., et al. (1995). Demonstrable cardiac reinnervation after human heart transplantation by carotid baroreflex modulation of RR interval.
Circulation 92, 2895-2903. doi: 10.1161/01.CIR.92.10.2895

Bernardi, L., Keller, F., Sanders, M., Reddy, P. S., Griffith, B., Meno, F., et al. (1989). Respiratory sinus arrhythmia in the denervated human heart. J. Appl. Physiol. 67, 1447-1455

Bezerra, S. M., dos Santos, C. M., Moreira, E. D., Krieger, E. M., and Michelini, L. C. (2001). Chronic AT(1) receptor blockade alters autonomic balance and sympathetic responses in hypertension. Hypertension 38, 569-575. doi: 10.1161/hy09t1.095393

Bigger, J. T. Jr., Fleiss, J. L., Steinman, R. C., Rolnitzky, L. M., Kleiger, R. E., and Rottman, J. N. (1992). Frequency domain measures of heart period variability and mortality after myocardial infarction. Circulation 85, 164-171. doi: 10.1161/01.CIR.85.1.164

Borst, C., Wieling, W., van Brederode, J. F., Hond, A., de Rijk, L. G., and Dunning, A. J. (1982). Mechanisms of initial heart rate response to postural change. Am. J. Physiol. 243, H676-H681.

Brennan, M., Palaniswami, M., and Kamen, P. (2001). Do existing measures of Poincaré plot geometry reflect nonlinear features of heart rate variability? IEEE Trans. Biomed Eng. 48, 1342-1347. doi: 10.1109/ 10.959330

Campos, L. A., Cipolla-Neto, J., Amaral, F. G., Michelini, L. C., Bader, M., and Baltatu, O. C. (2013a). The Angiotensinmelatonin axis. Int. J. Hypertens. 2013, 521783. doi: 10.1155/2013/ 521783

Campos, L. A., Cipolla-Neto, J., and Michelini, L. C. (2013b). Melatonin modulates baroreflex control via area postrema. Brain Behav. 3, 171-177. doi: 10.1002/brb3.123

Campos, L. A., Couto, A. S., Iliescu, R., Santos, J. A., Santos, R. A., Ganten, D., et al. (2004). Differential regulation of central vasopressin receptors in transgenic rats with low brain angiotensinogen. Regul. Pept. 119, 177-182. doi: 10.1016/j.regpep.2004.02.001

Campos, L. A., Iliescu, R., Fontes, M. A., Schlegel, W. P., Bader, M., and Baltatu, O. C. (2006a). Enhanced isoproterenol-induced

(CENTRO-07-ST24-FEDER-002031, co-funded by QREN, "Mais Centro" program). Susana Brás acknowledges the postdoctoral grant by Cloud Thinking Project (ref. BPD/DETI/5259/2013). Sónia Gouveia acknowledges the postdoctoral grant by FCT (ref. SFRH/BPD/87037/2012).

cardiac hypertrophy in transgenic rats with low brain angiotensinogen. Am. J. Physiol. Heart Circ. Physiol. 291, H2371-H2376. doi: 10.1152/ajpheart.01145.2005

Campos, L. A., Plehm, R., CipollaNeto, J., Bader, M., and Baltatu, O. C. (2006b). Altered circadian rhythm reentrainment to light phase shifts in rats with low levels of brain angiotensinogen. Am. J. Physiol. Regul. Integr. Comp. Physiol. 290, R1122-R1127. doi: 10.1152/ajpregu.00703.2005

Carvalho, H. S., Junqueira, L. F., and Souza-Neto, J. (2002). A computerized fuzzy logic system for evaluation of the cardiovascular autonomic function based on multiple functional tests. Comput. Cardiol. 29, 173-176. doi: 10.1109/CIC.2002.1166735

Cooke, W. H., Cox, J. F., Diedrich, A. M., Taylor, J. A., Beightol, L. A., Ames J. E., et al. (1998). Controlled breathing protocols probe human autonomic cardiovascular rhythms. Am. J. Physiol. 274, H709-H718.

Cooke, W. H., Hoag, J. B., Crossman, A. A., Kuusela, T. A., Tahvanainen, K. U., and Eckberg, D. L. (1999). Human responses to upright tilt: a window on central autonomic integration. J. Physiol. 517(Pt 2), 617-628. doi: 10.1111/j.1469-7793.1999.0617t.x

Dash, S., Chon, K. H., Lu, S., and Raeder, E. A. (2009). Automatic real time detection of atrial fibrillation. Ann. Biomed. Eng. 37, 1701-1709. doi: 10.1007/s10439-009-9740-z

DeGiorgio, C. M., Miller, P., Meymandi, S., Chin, A., Epps, J., Gordon, S., et al. (2010). RMSSD, a measure of vagusmediated heart rate variability, is associated with risk factors for SUDEP: the SUDEP-7 Inventory. Epilepsy Behav. 19, 78-81. doi: 10.1016/j.yebeh.2010.06.011

Ewing, D. J., Neilson, J. M., Shapiro, C. M., Stewart, J. A., and Reid, W. (1991). Twenty four hour heart rate variability: effects of posture, sleep, and time of day in healthy controls and comparison with bedside tests of autonomic function in diabetic patients. Br. Heart J. 65, 239-244. doi: 10.1136/hrt.65.5.239

Gamelin, F. X., Berthoin, S., and Bosquet, L. (2006). Validity of the polar S810 heart rate monitor to measure R-R intervals at rest Med. Sci. Sports Exerc. 38, 887-893. doi: $\quad 10.1249 / 01 . m s s .0000218135$. $79476.9 \mathrm{c}$

Gieraltowski, J., Hoyer, D., Tetschke, F., Nowack, S., Schneider, U., and Zebrowski, J. (2013). Development of multiscale complexity and multifractality of fetal heart rate variability. Auton. Neurosci. doi: 10.1016/j.autneu.2013.01.009

Gootman, P. M., and Cohen, M. I. (1970). Efferent splanchnic activity and systemic arterial pressure. Am. J. Physiol. 219, 897-903.

Gouveia, S., and Bras, S. (2012). "Exploring the use of fuzzy logic models to describe the relation between, SBP, and RR values," in Conference proceedings: Annual International Conference of the IEEE Engineering in Medicine, and Biology Society IEEE Engineering in Medicine, and Biology Society Conference 2012. 2827-2830.

Gouveia, S., Rocha, A. P., Laguna, P., and Lago, P. (2009). Time domain baroreflex sensitivity assessment by joint analysis of spontaneous SBP and RR series. Biomed. Signal. Proces. 4, 254-261. doi: 10.1016/j.bspc.2009.03.003

Gouveia, S., Rocha, A. P., Laguna, P., and van de Borne, P. (2010). Correlation between time domain baroreflex sensitivity and sympathetic nerve activity. Comput. Cardiol. 37, 5-8.

Grossi, E. (2005). Medical concepts related to individual risk are better explained with "plausibility" rather than "probability". BMC Cardiovasc. Disord. 5:31. doi: 10.1186/14712261-5-31

Harris, A. (2011). WHO: World Health Organization. Charleston Advisor 12, 54-56. doi: 10.5260/chara.12.4.54

Head, G. A., Lukoshkova, E. V., Burke, S. L., Malpas, S. C., Lambert, E. A., and Janssen, B. J. (2001). Comparing spectral and invasive estimates of baroreflex gain. IEEE Eng. Med. Biol. Mag. 20, 43-52. doi: 10.1109/51.917723

Hnatkova, K., Copie, X., Staunton, A., and Malik, M. (1995). Numeric processing of Lorenz plots of R-R intervals from long-term ECGs. Comparison with time-domain 
measures of heart rate variability for risk stratification after myocardial infarction. J. Electrocardiol. 28(Suppl.), 74-80. doi: 10.1016/ S0022-0736(95)80020-4

Hu, J., Gao, J., and Tung, W. W. (2009). Characterizing heart rate variability by scale-dependent Lyapunov exponent. Chaos 19, 028506. doi: 10.1063/1.3152007

Huikuri, H. V., Makikallio, T. H., and Perkiomaki, J. (2003). Measurement of heart rate variability by methods based on nonlinear dynamics. J. Electrocardiol. 36(Suppl.), 95-99. doi: $\quad 10.1016 /$ j.jelectrocard.2003. 09.021

Ishii, K., Kuwahara, M., Tsubone, H., and Sugano, S. (1996). Autonomic nervous function in mice and voles (Microtus arvalis): investigation by power spectral analysis of heart rate variability. Lab. Anim. 30, 359-364. doi: 10.1258/002367796780739880

Joaquim, L. F., Farah, V. M., Bernatova, I., Fazan, R. Jr., Grubbs, R., and Morris, M. (2004). Enhanced heart rate variability and baroreflex index after stress and cholinesterase inhibition in mice. Am.J. Physiol. Heart Circ. Physiol. 287, H251-H257. doi: 10.1152/ajpheart.01136.2003

Kamen, P. W., and Tonkin, A. M. (1995). Application of the Poincaré plot to heart rate variability: a new measure of functional status in heart failure. Aust. NZ. J. Med. 25, 18-26. doi: 10.1111/j.14455994.1995.tb00573.x

Kannathal, N., Acharya, U. R., Ng, E. Y., Krishnan, S. M., Min, L. C., and Laxminarayan, S. (2006). Cardiac health diagnosis using data fusion of cardiovascular and haemodynamic signals. Comput. Methods Prog. Biomed. 82, 87-96. doi: 10.1016/j.cmpb.2006.01.009

Kezdi, P., and Geller, E. (1968). Baroreceptor control of postganglionic sympathetic nerve discharge. Am. J. Physiol. 214, 427-435.

Kleiger, R. E., Miller, J. P., Bigger, J. T. Jr., and Moss, A. J. (1987). Decreased heart rate variability and its association with increased mortality after acute myocardial infarction. Am. J. Cardiol. 59, 256-262. doi: 10.1016/0002-9149(87)90795-8

Kovacic, Z., and Bogdan, S. (2006). Fuzzy Controller Design, Theory and Applications. Boca Raton, FL: Taylor and Francis Group.

Kuusela, T. A., Kaila, T. J., and Kahonen, M. (2003). Fine structure of the lowfrequency spectra of heart rate and blood pressure. BMC Physiol. 3:11. doi: 10.1186/1472-6793-3-11

La Rovere, M. T., Pinna, G. D., and Raczak, G. (2008). Baroreflex sensitivity: measurement and clinical implications. Ann. Noninvasive Electrocardiol. 13, 191-207. doi: 10.1111/j.1542-474X.2008.00219.x

Lee, J. W., Figeys, D., and Vasilescu, J. (2007). Biomarker assay translation from discovery to clinical studies in cancer drug development: quantification of emerging protein biomarkers. Adv. Cancer Res. 96, 269-298. doi: 10.1016/S0065230X(06)96010-2

Lerma, C., Infante, O., Perez-Grovas, H., and Jose, M. V. (2003) Poincare plot indexes of heart rate variability capture dynamic adaptations after haemodialysis in chronic renal failure patients. Clin. Physiol. Func. Imag. 23, 72-80. doi: 10.1046/j.1475-097X.2003.00466.x

Levin, A. B. (1966). A simple test of cardiac function based upon the heart rate changes induced by the Valsalva maneuver. Am. J. Cardiol. 18, 90-99. doi: 10.1016/0002-9149(66)90200-1

Lin, C. T., Liu, S. H., Wang, J. J., and Wen, Z. C. (2003). Reduction of interference in oscillometric arterial blood pressure measurement using fuzzy logic. IEEE Trans. Biomed. Eng. 50, 432-441. doi: 10.1109/TBME.2003.809502

Liu, J., McKenna, T. M., Gribok, A., Beidleman, B. A., Tharion, W. J. and Reifman, J. (2008). A fuzzy logic algorithm to assign confidence levels to heart and respiratory rate time series. Physiol. Meas. 29, 81-94. doi: 10.1088/0967-3334/29/1/006

Low, P. A. (1993). Autonomic nervous system function. J. Clin. Neurophysiol. 10, 14-27. doi: 10.1097/00004691-19930100000003

Malik, M., and Camm, A. J. (1993). Components of heart rate variability-what they really mean and what we really measure. Am. J. Cardiol. 72, 821-822. doi: 10.1016/0002-9149(93)91070-X

Malik, M., Farrell, T., Cripps, T., and Camm, A. J. (1989). Heart rate variability in relation to prognosis after myocardial infarction: selection of optimal processing techniques. Eur. Heart J. 10, 1060-1074.

Mancini, E., Mambelli, E., Irpinia, M., Gabrielli, D., Cascone, C., Conte, F., et al. (2007). Prevention of dialysis hypotension episodes using fuzzy logic control system. Nephrol. Dial. Transpl. 22, 1420-1427. doi: 10.1093/ndt/gfl799

Meier, R., Nieuwland, J., Zbinden, A. M., and Hacisalihzade, S. S. (1992). Fuzzy logic control of human blood pressure during anesthesia. IEEE Control Syst. 12, 12-17. doi: $10.1109 / 37.168811$
Nolan, J., Batin, P. D., Andrews, R. Lindsay, S. J., Brooksby, P., Mullen, M., et al. (1998). Prospective study of heart rate variability and mortality in chronic heart failure: results of the United Kingdom heart failure evaluation and assessment of risk trial (UK-heart) Circulation 98, 1510-1516. doi: 10.1161/01.CIR.98.15.1510

Nunes, C. S., and Amorim, P. (2008) "A neuro-fuzzy approach for predicting hemodynamic responses during anesthesia," in Conference proceedings: Annual International Conference of the IEEE Engineering in Medicine and Biology Society IEEE Engineering in Medicine and Biology Society Conference 2008, 5814-5817.

Oppenheim, A. V., and Schafer, R. W. (1975). Digital Signal Processing. Englewood Cliffs, NJ: Prentice Hall.

Ori, Z., Monir, G., Weiss, J., Sayhouni, X., and Singer, D. H. (1992). Heart rate variability. Frequency domain analysis. Cardiol. Clin. 10, 499-537.

Pagani, M., Lombardi, F., Guzzetti, S., Rimoldi, O., Furlan, R., Pizzinelli, P., et al, (1986). Power spectral analysis of heart rate and arterial pressure variabilities as a marker of sympatho-vagal interaction in man and conscious dog. Circulation 59, 178-193. doi: 10.1161/01.RES.59.2.178

Peng, C. K., Havlin, S., Stanley, H. E., and Goldberger, A. L. (1995). Quantification of scaling exponents and crossover phenomena in nonstationary heartbeat time series. Chaos 5, 82-87. doi 10.1063/1.166141

Petkovic, D., Cojbasic, Z., and Lukic, S. (2013). Adaptive neuro fuzzy selection of heart rate variability parameters affected by autonomic nervous system. Expert Syst. Appl. 40, 4490-4495. doi: 10.1016/j.eswa.2013.01.055

Pincus, S. M. (1991). Approximate entropy as a measure of system complexity. Proc. Natl. Acad. Sci. U.S.A. 88, 2297-2301. doi: 10.1073/pnas.88.6.2297

Porta, A., Catai, A. M., Takahashi, A. C., Magagnin, V., Bassani, T., Tobaldini, E., et al. (2011). Causal relationships between heart period and systolic arterial pressure during graded head-up tilt. Am. J. Physiol. Regul. Integr. Comp. Physiol. 300, R378-R386. doi: 10.1152/ajpregu.00553.2010

Rajendra Acharya, U., Paul Joseph, K., Kannathal, N., Lim, C. M., and Suri, J. S. (2006). Heart rate variability: a review. Med. Biol. Eng. Comput. 44, 1031-1051. doi: 10.1007/s11517-006-0119-0
Ramirez-Villegas, J. F., Lam-Espinosa, E., Ramirez-Moreno, D. F., Calvo-Echeverry, P. C., and Agredo-Rodriguez, W. (2011). Heart rate variability dynamics for the prognosis of cardiovascular risk. PLOS ONE 6, e17060. doi: 10.1371/journal.pone.0017060

Ryan, S. M., Goldberger, A. L., Pincus, S. M., Mietus, J., and Lipsitz, L. A. (1994). Gender- and age-related differences in heart rate dynamics: are women more complex than men? J. Am. Coll. Cardiol. 24, 1700-1707. doi: 10.1016/0735-1097(94)90177-5

Sadegh-Zadeh, K. (1999). Advances in fuzzy theory. Artif. Intell. Med. 15, 309-323. doi: 10.1016/S09333657(98)00060-8

Sassi, R., Signorini, M. G., and Cerutti, S. (2009). Multifractality and heart rate variability. Chaos 19, 028507. doi: 10.1063/1.3152223

Shields, R. W. Jr. (2009). Heart rate variability with deep breathing as a clinical test of cardiovagal function. Cleve. Clin. J. Med. 76(Suppl. 2), S37-S40. doi: 10.3949/ccjm.76.s2.08

Signorini, M. G., Margenes, G., Cerutti, S., and Arduini, D. (2003). Linear and nonlinear parameters for the analysis of fetal heart rate signal from cardiotocographic recordings. IEEE Trans. Biomed. Eng. 50, 365-374. doi: 10.1109/TBME.2003.808824

Silva, G. J., Ushizima, M. R., Lessa, P. S., Cardoso, L., Drager, L. F., Atala, M. M., et al. (2009). Critical analysis of autoregressive and fast Fourier transform markers of cardiovascular variability in rats and humans. Braz. J. Med. Biol. Res.42, 386-396. doi: 10.1590/ S0100-879X2009000400012

Silvani, A., Grimaldi, D., Vandi, S., Barletta, G., Vetrugno, R., Provini, F., et al. (2008). Sleep-dependent changes in the coupling between heart period and blood pressure in human subjects. Am. J. Physiol. Regul. Integr. Comp. Physiol. 294, R1686-R1692. doi: 10.1152/ajpregu.00756.2007

Silvani, A., Magosso, E., Bastianini, S., Lenzi, P., and Ursino, M. (2011). Mathematical modeling of cardiovascular coupling: central autonomic commands and baroreflex control. Auton. Neurosci. Basic Clin. 162, 66-71. doi: 10.1016/j.autneu.2011.04.003

Sundkvist, G., Lilja, B., and Almér, L. O. (1982). Deep breathing, Valsalva, and tilt table tests in diabetics with and without symptoms of autonomic neuropathy. Acta Med. Scand. 211, 369-373. doi: 10.1111/j.0954 6820.1982.tb01964.x 
Task force. (1996). Heart rate variability: standards of measurement, physiological interpretation and clinical use. Task force of the european society of cardiology and the north american society of pacing and electrophysiology. Circulation 93, 1043-1065. doi: 10.1161/01.CIR.93.5.1043

Taylor, J. A., Carr, D. L., Myers, C. W., and Eckberg, D. L. (1998). Mechanisms underlying very-lowfrequency RR-interval oscillations in humans. Circulation 98, 547-555. doi: 10.1161/01.CIR. 98.6.547

Tsipouras, M. G., Exarchos, T. P., and Fotiadis, D. I. (2007). "Integration of global and local knowledge for fuzzy expert system creation: application to arrhythmic beat classification," in Conference proceedings: Annual International Conference of the IEEE Engineering in Medicine and Biology Society IEEE Engineering in Medicine and Biology Society Conference 2007, 3840-3843. doi: 10.1109/IEMBS.2007.4353170

Tulppo, M. P., Makikallio, T. H., Takala, T. E., Seppanen, T., and Huikuri, H. V. (1996). Quantitative beat-tobeat analysis of heart rate dynamics during exercise. Am. J. Physiol. 271, H244-H252.

Ushizima, M. R., Moreira, E. D., Costa, E. T., Castiglioni, P., Krieger, E. M., Di Rienzo, M., et al. (2001). Effects of sinoaortic denervation on blood pressure, pulse interval, sympathetic nerve activity and diaphragmatic electromyogram variability in conscious rats. Comput. Cardiol. 28, 485-488.

Vanderlei, L. C., Pastre, C. M., Freitas, I. F. Jr., and Godoy, M. F. (2010). Geometric indexes of heart rate variability in obese and eutrophic children. Arq. Bras. Cardiol. 95, 35-40. doi: 10.1590/S0066782X2010005000082

Vanderlei, L. C., Pastre, C. M., Hoshi, R. A., Carvalho, T. D., and Godoy, M. F. (2009). Basic notions of heart rate variability and its clinical applicability. Rev. Bras. Cir. Cardiovasc. 24, 205-217. doi: 10.1590/S010276382009000200018

Voss, A., Schroeder, R., Truebner, S., Goernig, M., Figulla, H. R., and Schirdewan, A. (2007). Comparison of nonlinear methods symbolic dynamics, detrended fluctuation, and Poincare plot analysis in risk stratification in patients with dilated cardiomyopathy. Chaos 17, 015120. doi: 10.1063/1.2404633

Westerhof, B. E., Gisolf, J., Karemaker, J. M., Wesseling, K. H., Secher, N. H., and van Lieshout, J. J. (2006). Time course analysis of baroreflex sensitivity during postural stress. Am. J. Physiol. Heart Circ. Physiol. 291, H2864-H2874. doi: 10.1152/ajpheart.01024.2005

Wheeler, T., and Watkins, P. J. (1973). Cardiac denervation in diabetes. Br. Med. J. 4, 584-586. doi: 10.1136/bmj.4.5892.584

Woo, M. A., Stevenson, W. G., Moser, D. K., Trelease, R. B., and Harper, R. M. (1992). Patterns of beatto-beat heart rate variability in advanced heart failure. Am. Heart J. 123, 704-710. doi: 10.1016/00028703(92)90510-3

World Health Organization. (2011). Global Status Report on Noncommunicable Diseases, 2010. Geneva: World Health Organization.

Zadeh, L. A. (1975). Fuzzy logic and approximate reasoning. Synthese 30, 407-428. doi: 10.1007/BF00485052

Conflict of Interest Statement: The authors declare that the research was conducted in the absence of any commercial or financial relationships that could be construed as a potential conflict of interest.

Received: 30 July 2013; paper pending published: 12 August 2013; accepted: 17 September 2013; published online: 07 October 2013.

Citation: Campos LA, Pereira VL Jr, Muralikrishna A, Albarwani S, Brás S and Gouveia S (2013) Mathematical biomarkers for the autonomic regulation of cardiovascular system. Front. Physiol. 4:279. doi: 10.3389/fphys.2013.00279

This article was submitted to Integrative Physiology, a section of the journal Frontiers in Physiology.

Copyright (c) 2013 Campos, Pereira, Muralikrishna, Albarwani, Brás and Gouveia. This is an open-access article distributed under the terms of the Creative Commons Attribution License (CC BY). The use, distribution or reproduction in other forums is permitted, provided the original author(s) or licensor are credited and that the original publication in this journal is cited, in accordance with accepted academic practice. No use, distribution or reproduction is permitted which does not comply with these terms. 